\title{
Comparison of two transconjunctival and transcutaneous approaches in the incidence of scleral show during lower eyelid blepharoplasty
}

\author{
Hooman Hooshangi ${ }^{1}$, Mehdi Sezavar ${ }^{2 *}$, Behnam Bohluli ${ }^{3}$, Farzin Sarkarat ${ }^{3}$, Fatemeh \\ Nasrollahi ${ }^{1}$ and Sina Seyedabbaszadeh ${ }^{1}$ \\ ${ }^{1}$ Resident of Oral and Maxillofacial Surgery, Islamic Azad University, Dental Branch, Tehran, Iran \\ ${ }^{2}$ Assistant Professor, Department of Oral and Maxillofacial Surgery, Islamic Azad University, Dental Branch, \\ Tehran, Iran \\ ${ }^{3}$ Associated Professor, Department of Oral and Maxillofacial Surgery, Islamic Azad University, Dental \\ Branch, Tehran, Iran
}

\begin{abstract}
Despite its effectiveness for the facial rejuvenation, blepharoplasty has been associated with several adverse effects. One of the most common side effects of this surgery is eyelid malposition and scleral show which is not suitable from esthetic point of view. Although some techniques have been used for the blepharoplasty of the lower eyelid, the effect of these techniques has not been definitely identified in the incidence of scleral show. The present study compared the effects of lower eyelid blepharoplasty with transconjunctival and transcutaneous approaches in the incidence of scleral show in patients. 22 candidates of the lower eyelid blepharoplasty were studied. The patients had no history of systemic disease, previous lower eyelid blepharoplasty, trauma or dry eye syndrome. The patients were randomly assigned in 2 methods of transconjunctival and transcutaneous approaches. The surgeries were perform on 2 eyelids of the left and right sides and in total, 44 eyelids were subjected to blepharoplasty surgery in both sides of the patients in 2 approaches. The incidence of scleral show in both modalities was studied 2, 4 and 6 months post-surgery. In this study, 22 patients were included for eyelid blepharoplasty (44 eyelids). In the transconjuctival approach, 4 males and 7 females with average age of the 57.09 years old were included. For transcutaneous approach, 5 males and 5 females average age of the 56.18 years old were included. According to the results, no scleral was showed using transconjuctival approach after 2 months. Two months after transcutaneous approach only 2 (9\%) scleral was observed (P<0.8). No
\end{abstract}

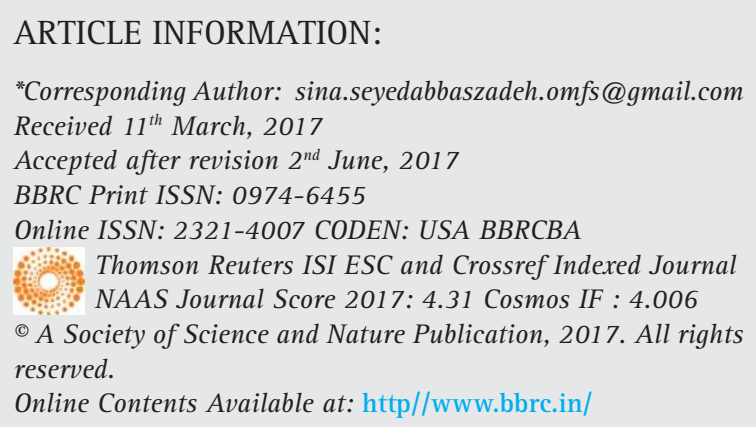


significant difference detected in scleral using transconjuctival or transcutaneous operations after 4 months, however only $2(9 \%)$ scleral was observed using transcutaneous approach $(\mathrm{P}<0.8)$. The same results observed after 6 months follow-up period $(\mathrm{P}<0.8)$.Despite insignificant differences between 2 lower eyelid blepharoplasty approaches regarding the scleral show incidence, using transconjunctival technique was recommended for the lower eyelid blepharoplasty in order to decrease the incidence of the scleral show.

KEY WORDS: LOWER EYELID BLEPHAROPLASTY, TRANSCUTANEOUS, TRANSCONJUNCTIVAL, SCLERAL SHOW

\section{INTRODUCTION}

Blepharoplasty remains one of the most common contemporary facial surgical procedures performed. While in oculo-facial plastic surgery functional upper eyelid blepharoplasty still encompasses the majority of the specialties procedures, cosmetic upper and lower blepharoplasty are rapidly increasing in number.The skin-muscle flap approach to lower blepharoplasty was popularized in the 1970s. It remained the most generally used method because of e fast, effective and widely applicable (Hidalgo, 2011; Massry and Hartstein, 2012). Post blepharoplasty treatment of lower eyelid retraction is challenging (Yoo et al. 2013). Several methods introduced to improve lower eyelid blepharoplasty outcome (Schwarcz et al. 2016, Kossler et al. 2017).

Transcutaneous lower eyelid blepharoplasty with fat excision has been the paradigm for addressing the undesirable sequelae of the aging eye (Griffin et al. 2014). This is eyelid contour and no changes in eyelid position when performed in a conservative fashion. Transcutaneous is a time-tested method that achieves satisfactory cosmetic results (Garcia and McCollough, 2006). The latest evolution of the transcutaneous lower eyelid blepharoplasty is a skin flap or skin muscle flap method in which the incision is placed inferiorly to the tarsal margin (Freeman, 2000). The transconjunctival approach was primary described in 1924 to the lower orbital fat and the inferior orbital surface (Korchia et al. 2003). Transconjunctival lower eyelid blepharoplasty is most acceptable as a safe and effective method to eliminate herniated orbital fat from the lower eyelid and avoiding the complications of a cutaneous incision (Belinsky et al. 2015).

The transconjunctival approach avoids numerous complications of the classic transcutaneous lower blepharoplasty (Kossler et al. 2017). It is reported the limitations of the transcutaneous approach was improved by the transconjunctival approach (LoPiccolo et al. 2013). The transconjunctival approach has gained popularity over the transcutaneous approaches because of its inconspicuous scar and decreased risk of postoperative ectropion and scleral show (Salhi and Cordoba, 2015).

Scleral show is one of the main complications of lower blepharoplasty which may result to manifest as lack of definition of the lateral part of the eyes and eyelid retraction (Pascali et al. 2015). Lower eyelid retraction is known for the existence of scleral show (which each patient stated (Griffin et al. 2014). Scleral show is one of the most feared complications of lower blepharoplasty surgery. The attendant scleral show and rounding of the eye are poorly tolerated by patients from a cosmetic and often functional stand-point (Griffin et al. 2014). The surgical correction of postsurgical eyelid retraction can be challenging as well as frustrating (Yoo et al. 2014).

Despite its effectiveness for the facial rejuvenation, blepharoplasty has been associated with several adverse effects. One of the most common side effects of this surgery is eyelid malposition and scleral show which is not suitable from esthetic point of view. Although some techniques have been used for the blepharoplasty of the lower eyelid, the effect of these techniques has not been definitely identified in the incidence of scleral show. So, the current study was compared the effects of lower eyelid blepharoplasty with transconjunctival and transcutaneous approaches in the incidence of scleral show in patients.

\section{MATERIAL AND METHODS}

In a prospective randomized clinical trial, 22 candidates of the lower eyelid blepharoplasty in patients referred to two private clinics and Bu Ali hospital at 2015. The patients had no history of systemic disease, previous lower eyelid blepharoplasty, trauma or dry eye syndrome. The patients were randomly assigned in 2 methods of transconjunctival and transcutaneous approaches. The surgeries were perform on 2 eyelids of the left and right sides and in total, 44 eyelids were subjected to blepharoplasty surgery in both sides of the patients in 2 approaches. The incidence of scleral show in both modalities was studied using standard photographs of patients taken before the surgery and 2, 4 and 6 months post-surgery. The frequency of the scleral show was statistically analyzed by exact fisher test in two approaches in different time intervals.

\section{RESULTS}

In this study, 22 patients were included for eyelid blepharoplasty (44 eyelids). In the transconjuctival approach, 4 
Table 1. the demographic information of the patients based on the surgery operation

\begin{tabular}{|l|c|c|c|c|c|c|} 
& \multicolumn{2}{|c|}{ Sex } & \multicolumn{2}{c|}{ Location } & age & Following period (month) \\
\hline & Male & Female & Left & Right & & \\
\hline Transconjuctival & 4 & 7 & 11 & 11 & 57.09 & 6 \\
\hline Transcutaneous & 5 & 6 & 11 & 11 & 56.18 & 6 \\
\hline
\end{tabular}

males and 7 females with average age of the 57.09 years old were included. For transcutaneous approach, 5 males and 5 females average age of the 56.18 years old were included. Then all patients were followed-up 6 months for possible scleral show (table 1).

The results of the scleral show at 2-6 months post transconjuctival or transcutaneous operations are presented in table 2. According to the results, no scleral was showed using transconjuctival approach after 2 months. Two months after transcutaneous approach only 2 (9\%) scleral was observed $(\mathrm{P}<0.8)$. No significant difference detected in scleral using transconjuctival or transcutaneous operations after 4 months, however only 2 (9\%) scleral was observed using transcutaneous approach $(\mathrm{P}<0.8)$. The same results observed after 6 months follow-up period $(\mathrm{P}<0.8)$.

\section{DISCUSSION}

Eyelid tone, position and scleral show have been the most common limiting aspects of lower blepharoplasty. Dysfunction of the lateral canthal tendon can be caused by aging, iatrogenic damage, and so on (Sacchidanand et al. 2012). Lower eyelid retraction might occur by dynamic imbalance in the lateral suspension system of the lower eyelid, lack of elasticity of mid facial soft tissue and etc. (Oestreicher and Mehta, 2012). Base on the results of the current study, no scleral was showed using transconjuctival approach after 2 months. Two months after transcutaneous approach only 2 (9\%) scleral was observed. No significant difference detected in scleral using transconjuctival or transcutaneous operations after 4 months, however only 2 (9\%) scleral was observed using transcutaneous approach. The same results observed after 6 months follow-up period. In this regard, Bernardino et al. (2016) reported there was improvement in all 16 female and 2 male patients in the appearance of increased the lower eyelid and reduction in skin and lateral ligament laxity. Also, eyelid retraction, ectropion, unaesthetic scars, diplopia and remnant fat bags were not observed (Bernardino et al. 2016).

During the 1998-2008 in 2400 patients underwent lower blepharoplasty Transconjunctival blepharoplasty was the first choice for primary eyelid bags. Transcutaneous lower blepharoplasty (skin flap or skin-muscle flap procedures) is indicated for the senile eyelid with excess skin and muscles (Guo et al. 2010).Both transconjunctical and transcutaneous approaches is applied for upper and lower lid blepharoplasty. Even though transcutaneous upper and lower eyelid surgery and transconjunctival lower lid procedures are popular with facial plastic surgeons, transconjunctival upper blepharoplasty is new technique with limited indications (Jacono et al. 2001). These researchers suggested the transconjunctival lower lid blepharoplasty as it circumvents the risk of lower eyelid retraction associated with the transcutaneous approach (Jacono et al. 2001). A lower rate of complications reported via the transconjunctival approach, with greater patient satisfaction (Rancati et al. 2015).

The transconjunctival and approaches were preferred in 42 and $58 \%$ (Rancati et al. 2015). In transconjunctival versus transcutaneous lower eyelid blepharoplasty (Netscher et al. 1995) in 6 months follow-up reported 10 consecutive patients presented for blepharoplasty, and in all patients the transcutaneous subciliary musculocutaneous flap approach was used on the left lower eyelid and the transconjunctival preseptal approach was used on the right. No significant variance on the 2 sides $(0.60$ on the left and 0.68 on the right) (Netscher et al. 1995). No significant difference reported in lower eyelid position change between the transconjunctival lower blepharoplasty with versus without a skin pinch groups. Using the transcutaneous approach, the presented infection

\begin{tabular}{|c|c|c|c|c|c|c|}
\hline & \multicolumn{2}{|c|}{ Follow (2 months) } & \multicolumn{2}{|c|}{ Follow (4 months) } & \multicolumn{2}{|c|}{ Follow (6 months) } \\
\hline & \multicolumn{6}{|c|}{ scleral show } \\
\hline & No & Yes & No & Yes & No & Yes \\
\hline Transconjuctival & $22(100 \%)$ & $0(0 \%)$ & $22(100 \%)$ & $0(0 \%)$ & $22(100 \%)$ & $0(0 \%)$ \\
\hline Transcutaneous & $20(91 \%)$ & $2(9 \%)$ & $20(91 \%)$ & $2(9 \%)$ & 20 (91\%) & $2(9 \%)$ \\
\hline Fisher's P value & \multicolumn{2}{|c|}{$<0.8$} & \multicolumn{2}{|c|}{$<0.8$} & \multicolumn{2}{|c|}{$<0.8$} \\
\hline
\end{tabular}


Hooman Hooshangi et al.

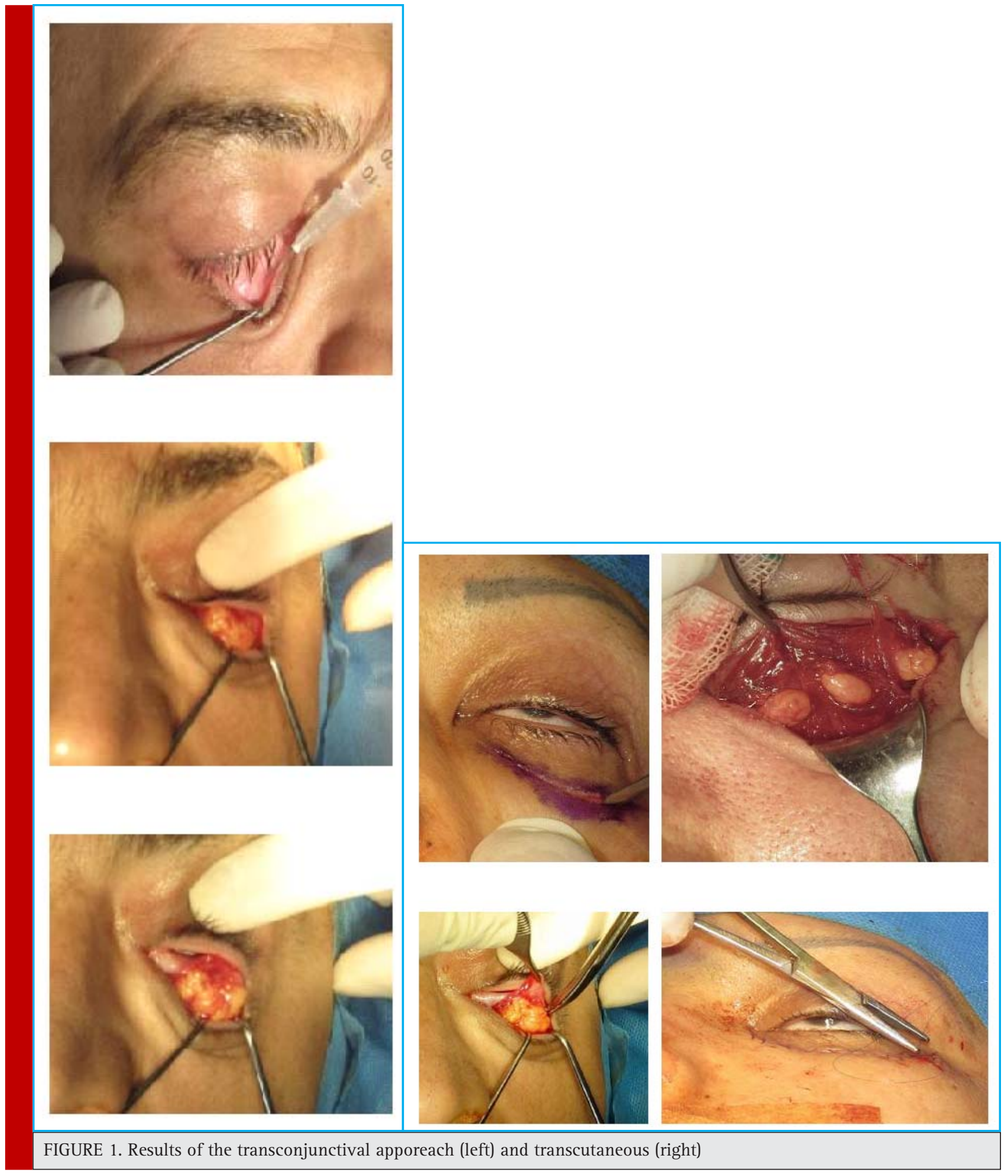



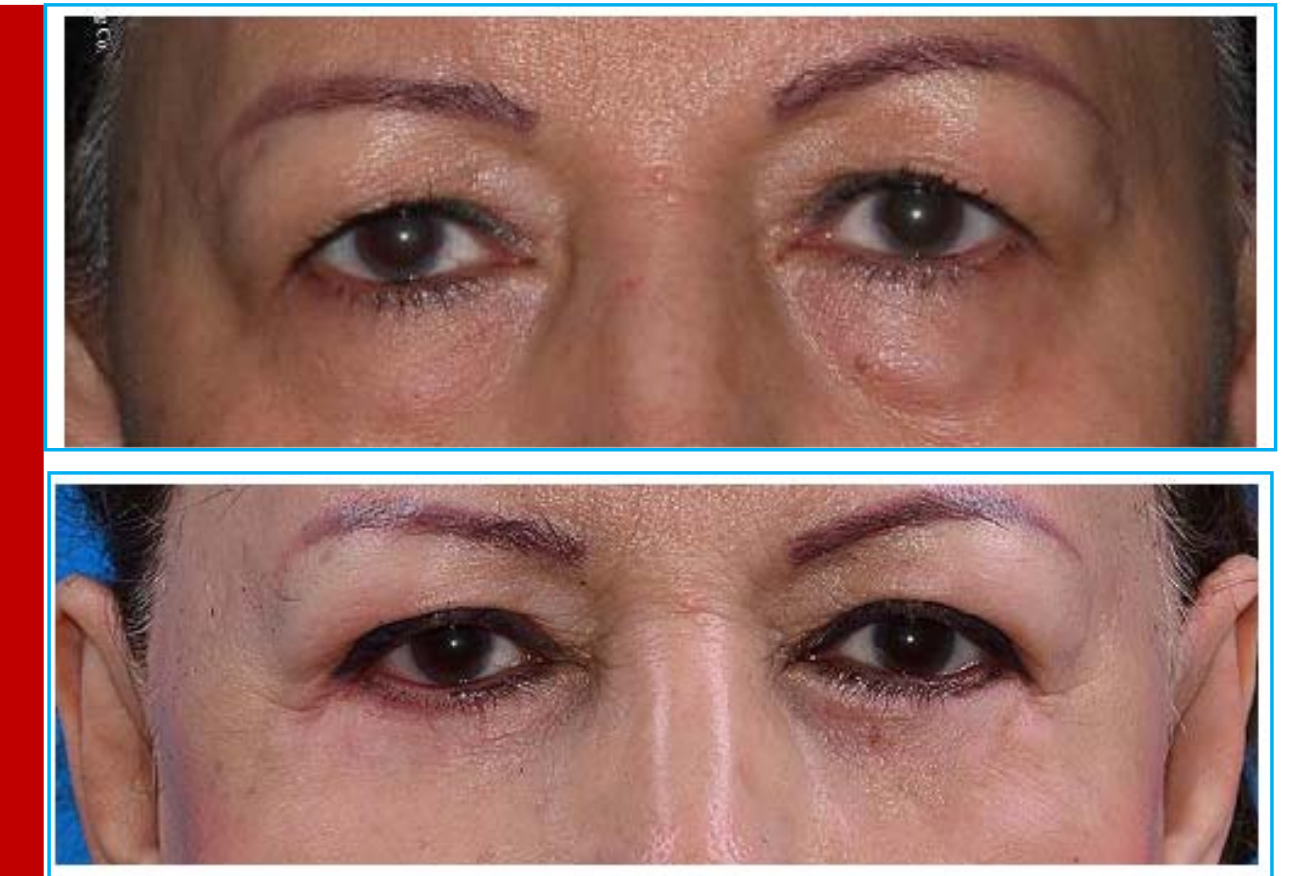

FIGURE 2. Results of the transconjunctival apporeach before and after the surgery

(2\%), scleral show (2\%) and insufficient skin resection (1\%) was reported (Hidalgo, 2011). The transconjunctival approach is an ideal procedure due to its simplicity and less traumatic effect, except in the lower eyelid weakness and surgical resolution (Collar et al. 2013). In conclu- sion, despite insignificant differences between 2 lower eyelid blepharoplasty approaches regarding the scleral show incidence, using transconjunctival technique was recommended for the lower eyelid blepharoplasty in order to decrease the incidence of the scleral show.

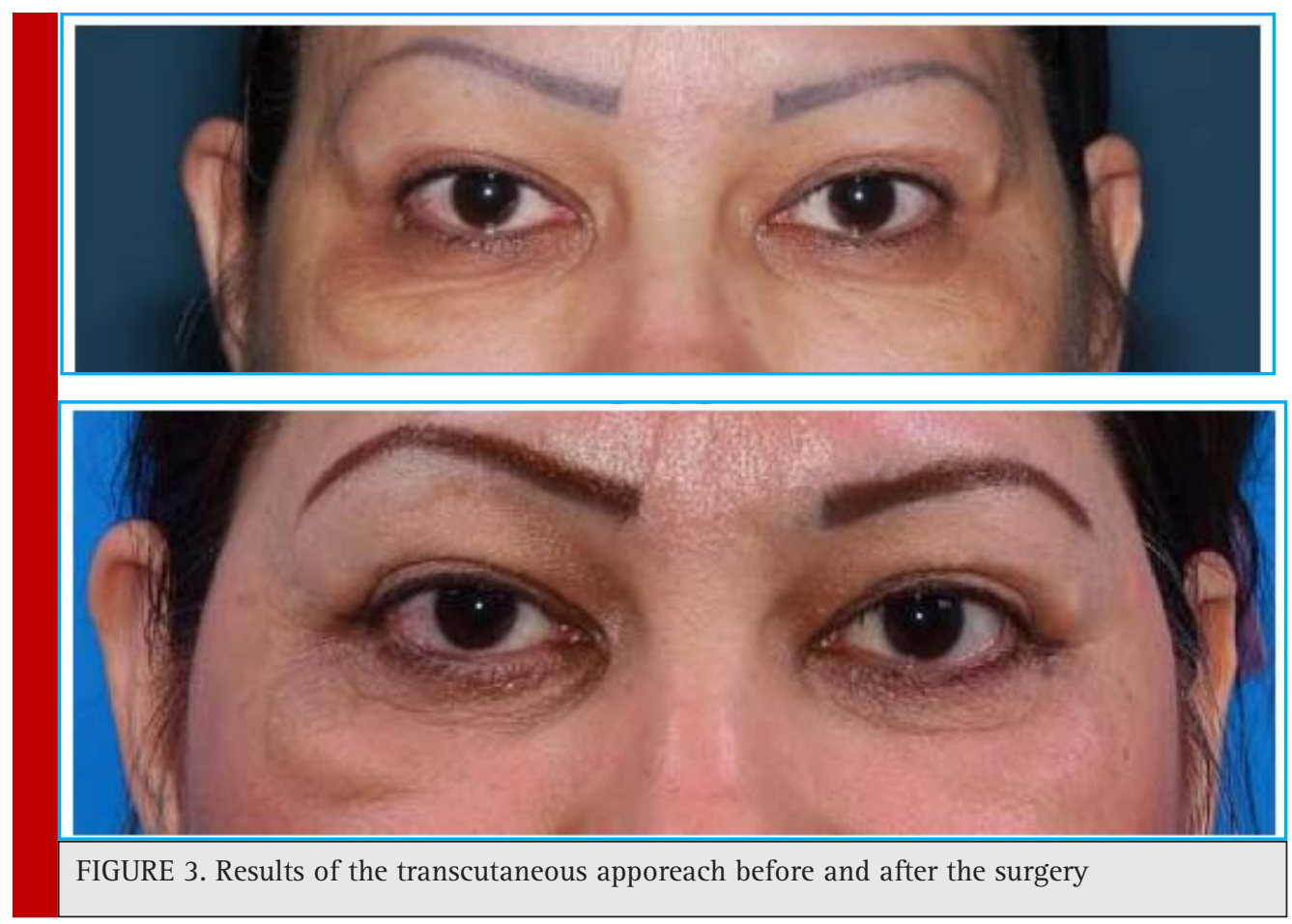




\section{REFERENCES}

Belinsky I, Patel P, Charles NC, Lisman RD. 2015. Ointment granulomas following sutureless transconjunctival blepharoplasty: diagnosis and management. Ophthal Plast Reconstr Surg;31:282-286

Bernardino IP. 2016. Transconjunctival lower blepharoplasty with skin resection and preservation of the orbicularis muscl. Rev. Bras. Cir. Plást. 31(1):74-81.

Collar, R.M., Lyford-Pike, S. and Byrne P. (2013) Algorithmic Approach to Lower Lid Blepharoplasty. Facial Plastic Surgery, 29, 32-39.

Freeman MS.2000.Transconjunctival sub-orbicularis oculi fat (SOOF) pad lift blepharoplasty.Arch Facial Plast Surg. 2: 16-21.

Garcia RE, McCollough E. G.2006. Transcutaneous lower eyelid blepharoplasty with fat excision. Arch Facial Plast Surg. 8:374-380.

Griffin G, Azizzadeh B, Massry GG.2014. New Insights Into Physical Findings Associated With Postblepharoplasty Lower Eyelid Retraction. Aesthetic Surgery Journal. 34: 995

Guo L, Bi H, Xue C, Li J, Yan C, Song J, Zhang M, Xing X. 2010. Comprehensive considerations in blepharoplasty in an Asian population: a 10-year experience. Aesth Plast Surg 34:466-474.

Hidalgo DA. 2011. An integrated approach to lower blepharoplasty. Plast. Reconstr. Surg.127: 386.

Jacono AA, Moscowitz B.2001. Transconjuctival versus Transcutaneous approach in upper and lower blepharoplasty. Facial Plast Surg 17(1):21-28.

Korchia D Braccini, F, Paris J Thomassin, JM. 2003. Transconjunctival approach in lower eyelid blepharoplasty. Can J Plast Surg 11(3):166-170.

Kossler AL, Peng GL, Yoo DB, Azizzadeh B, Massry GG. 2017. Current trends in upper and lower eyelid blepharoplasty among american society of ophthalmic plastic and reconstructive surgery members. Ophthal Plast Reconstr Surg;XX:00-00
LoPiccolo MC, Mahmoud BH, Liu A, et al.2013. Evaluation of orbi-cularis oculi muscle stripping on the cosmetic outcome of upper lid blepharoplasty: a randomized, controlled study. Dermatol Surg 39:739-43.

Massry GG, Hartstein ME. 2012.The lift and fill lower blepharoplasty. Ophthal Plast Reconstr Surg 28:213-8.

Netscher DT, Patrinely JR, Peltier M, Polsen C, Thornby J. 1995. Transconjunctival versus transcutaneous lower eyelid blepharoplasty: a prospective study. Plast Reconstr Surg ; 96(5):10531060.

Oestreicher J, Mehta S. 2012 Complications of blepharoplasty: prevention and management.Plast Surg Int. 2012: 252368.

Pascali M, Avantaggiato A, Brinci L, Cervelli V, Carinci F. 2015. Tarsal sling: an essential stitch to prevent scleral show in lower blepharoplasty. Aesthetic Surgery Journal 35(1) 11-19.

Rancati A, Jacovella P, Edoardo Zampieri A, Dorr J, Daniele M, Liedtke S, Rancati A. 2015. Lower blepharoplasty review: transconjunctival vs. transcutaneous approach. Modern Plast Surg 5:1-8.

Sacchidanand SA, Deepak HS, Vishal C, Revathy TN.2012 Transcutaneous blepharoplasty in blepharochalasis. J Cutan Aesthet Surg. 5:284-286.

Salhi S, Cordoba C. 2015. Distichiasis following transconjunctival approach to the inferior orbital rim and orbital floor. JPRAS Open 5 29-33.

Schwarcz R, Fezza JP, Jacono A, et al. 2016. Stop blaming the septum. Ophthal Plast Reconstr Surg 32:49-52.

Taban M, Taban M, Perry JD. 2008. Lower eyelid position after trans-conjunctival lower blepharoplasty with versus without a skin pinch. Ophthal Plast Reconstr Surg 24(1):7-9.

Yoo D, Griffin GG, Azizzadeh BA.2014. The minimally invasive orbicularis sparing lower eyelid recession. JAMA Arch Facial Plast Surg. 16:140-146.

Yoo DB, Peng GL, Massry GG.2013. Effacing the orbitoglabellar groove with transposed upper eyelid fat. Ophthal Plast Reconstr Surg 29:220-4. 